\title{
Cyclophosphamide-Induced Severe Acute Hyponatremic Encephalopathy in Patients with Breast Cancer: Report of Two Cases
}

\author{
Michelle Baker $^{\mathrm{a}} \quad$ Maurie Markman ${ }^{\mathrm{b}, \mathrm{c}}$ Jiaxin Niu ${ }^{\mathrm{b}}$ \\ Departments of a Pharmacy and ${ }^{b}$ Medical Oncology, Western Regional Medical Center, \\ Cancer Treatment Centers of America, Goodyear, Ariz., and 'Drexel University College of \\ Medicine, Philadelphia, Pa., USA
}

\section{Key Words}

Cyclophosphamide $\cdot$ Chemotherapy $\cdot$ Encephalopathy $\cdot$ Hyponatremia $\cdot$ Syndrome of inappropriate antidiuretic hormone secretion

\begin{abstract}
Background: Cyclophosphamide is an alkylating agent widely used in antineoplastic and immunosuppressive therapies. Symptomatic hyponatremia can be a rare but life-threatening complication in patients treated with cyclophosphamide. Case Presentations: We report 2 patients who presented with severe acute hyponatremic encephalopathy after receiving their first cycles of a low-dose cyclophosphamide-containing regimen for breast cancer. In case 1 , a 58-year-old female received the combination of docetaxel and cyclophosphamide, and in case 2, a 56-year-old female received the combination of doxorubicin and cyclophosphamide. Both patients recovered after correction of their serum sodium concentration without neurological deficits. Future cycles of chemotherapy were well tolerated without recurrence of hyponatremia after cyclophosphamide was discontinued from the respective regimens. Conclusion: Clinicians must always keep in mind that acute hyponatremic encephalopathy can be induced by low-dose cyclophosphamide.


Baker et al.: Cyclophosphamide-Induced Severe Acute Hyponatremic Encephalopathy in Patients with Breast Cancer: Report of Two Cases

\section{Background}

Cyclophosphamide is an alkylating agent used to treat a variety of malignancies and autoimmune disorders. The combination of cyclophosphamide with doxorobucin (AC) or docetaxel (TC) is widely used to treat breast cancer in both adjuvant and metastatic settings.

Cyclophosphamide is a prodrug and needs to be metabolized by the cytochrome P450 system to active metabolites that prevent cell division by cross-linking DNA strands and decreasing DNA synthesis. Transient nausea, vomiting, and myelosuppression are common side effects associated with high-dose cyclophosphamide. Hemorrhagic cystitis and hyponatremia are uncommon.

Chemotherapy-induced hyponatremia (serum sodium level $<135 \mathrm{mmol} / \mathrm{l}$ ) is a common electrolyte disorder encountered in clinical practice. Most cases are mild and asymptomatic and do not require medical intervention. By contrast, severe hyponatremia, defined as a serum sodium level $<120 \mathrm{mmol} / \mathrm{l}$, is rare but can cause significant morbidity and mortality.

Here we report 2 cases of severe acute hyponatremic encephalopathy in breast cancer patients after receiving their first cycle of chemotherapy containing low-dose cyclophosphamide.

\section{Case Presentations}

Case 1

A 58-year-old white female was originally diagnosed with invasive ductal carcinoma of the left breast in 2000. She underwent left lumpectomy and adjuvant radiation but declined adjuvant systemic therapy. In 2009, the patient was found to have in-breast recurrence and underwent a left mastectomy with immediate reconstruction. Unfortunately, again she declined chemotherapy or hormonal therapy.

The patient developed a right-sided pleural effusion in August 2012 with a biopsy of the pleura-based nodule revealing an estrogen receptor-positive, progesterone receptorpositive, human epithelial growth factor 2-negative metastatic adenocarcinoma, consistent with her primary breast cancer. She was started on hormone therapy with letrozole. Unfortunately, she experienced progressively worsening cough after approximately 3 months of treatment. Restaging confirmed progression of the disease with liver metastases.

Hormone therapy was discontinued and chemotherapy with the TC regimen was recommended. On October 24, 2012, the patient received standard intravenous premedications including palonosetron $0.25 \mathrm{mg}$, dexamethasone $12 \mathrm{mg}$, diphenhydramine $25 \mathrm{mg}$, and famotidine $20 \mathrm{mg}$, followed by docetaxel $130 \mathrm{mg}\left(75 \mathrm{mg} / \mathrm{m}^{2}\right)$ i.v. over $60 \mathrm{~min}$ and cyclophosphamide $1,040 \mathrm{mg}\left(600 \mathrm{mg} / \mathrm{m}^{2}\right.$, roughly $\left.15 \mathrm{mg} / \mathrm{kg}\right)$ i.v. over $60 \mathrm{~min}$. She tolerated this treatment reasonably well without any adverse reaction and received pegfilgrastim on the following day for prophylaxis of neutropenic fever.

Approximately 48-72 h after completing her first cycle, she started to experience worsening nausea, vomiting, and generalized weakness. The patient went to a local emergency room where she was diagnosed with hyponatremia (sodium $117 \mathrm{mmol} / \mathrm{l}$ ) secondary to dehydration. She received normal saline for rehydration as well as ondansetron for emesis and was discharged home. She developed a headache, experienced three falls, and became increasingly confused over the next 3 days. She returned to the emergency room for further workup and management. The patient was found to have stable vital signs with normal oxygenation on room air; however, she was notably lethargic with incoherent speech. She had no neurological deficits and the rest of the physical examinations were unremarkable. 
Baker et al.: Cyclophosphamide-Induced Severe Acute Hyponatremic Encephalopathy in Patients with Breast Cancer: Report of Two Cases

Laboratory studies revealed serum sodium $115 \mathrm{mmol} / \mathrm{l}$, potassium $3.9 \mathrm{mmol} / \mathrm{l}$, BUN 10 $\mathrm{mg} / \mathrm{dl}, \mathrm{Cr}$ 0.6/dl, serum osmolality $248 \mathrm{mOsm} / \mathrm{l}$ (284-304), and random urine osmolality $460 \mathrm{mOsm} / \mathrm{kg}$ (300-900). The brain computed tomography (CT) scan was unremarkable. Her altered mental status was attributed to her severe hyponatremia. As the patient's sodium was $138 \mathrm{mmol} / \mathrm{l}$ before chemotherapy, her hyponatremia was deemed to be due to syndrome of inappropriate antidiuretic hormone (SIADH) secretion, most likely secondary to cyclophosphamide. She was admitted to the hospital with free water restriction and conservative infusion of $0.9 \%$ saline 1 liter/day. Her mental status gradually returned to baseline as the sodium level improved. She was discharged home after 3 days without any neurological deficits following correction of her serum sodium. Cyclophosphamide was discontinued for the following cycles, and she tolerated docetaxel with the same premedications without recurrence of hyponatremia.

Case 2

A 56-year-old white female presented to our center in January 2013 with a widely metastatic breast cancer involving both breasts, lungs, liver, and bones. A biopsy confirmed the diagnosis of triple negative breast cancer. In light of the significant tumor burden, the patient was recommended to receive chemotherapy with doxorubicin $95 \mathrm{mg}\left(60 \mathrm{mg} / \mathrm{m}^{2}\right)$ i.v. over 10 min and cyclophosphamide $950 \mathrm{mg}\left(600 \mathrm{mg} / \mathrm{m}^{2}\right.$, roughly $\left.15 \mathrm{mg} / \mathrm{kg}\right)$ i.v. over $60 \mathrm{~min}$ (AC regimen). She received the same standard premedications as the patient above and tolerated the therapy well initially with only mild nausea and no vomiting.

Approximately $24 \mathrm{~h}$ after completing her first cycle, the patient presented at the clinic with the chief complaint of feeling fatigued and sleepy. She was found to be awake, oriented, and able to answer all assessment questions appropriately. She reported mild dyspnea, but her physical examination was unremarkable with an oxygen saturation over $95 \%$. Her vital signs were found to be stable. However, her level of consciousness started to decline shortly after her arrival and she was witnessed to have a generalized seizure with convulsions. Given the history of recent administration of narcotics including both methadone and oxycodone, narloxone was given, but the patient continued to be restless and unresponsive.

Although her airway was open, the patient developed hypoxia. She was placed on oxygen and admitted to the ICU for further management. Laboratory tests showed sodium 113 $\mathrm{mmol} / \mathrm{l}$, potassium $4.9 \mathrm{mmol} / \mathrm{l}$, and $\mathrm{Cr} 0.9 \mathrm{mg} / \mathrm{dl}$. Her brain CT scan was unremarkable, but the CT scan of the chest/abdomen/pelvis showed bilateral lower lobe consolidation and liver metastases. Her symptoms were described as metabolic encephalopathy resulting from severe hyponatremia secondary to cyclophosphamide. The diagnosis was based on her normal sodium level prior to chemotherapy. The patient received hypertonic saline, and after gradual correction of her hyponatremia to a sodium level $>130 \mathrm{mmol} / \mathrm{l}$, her mental status returned to baseline, and she was discharged home with a full recovery.

She received doxorubicin alone without administration of cyclophosphamide in the following cycles. The patient tolerated the adjusted regimen well without any evidence of mental status change or recurrence of hyponatremia.

\section{Discussion}

Severe hyponatremia is a serious, potentially life-threatening clinical diagnosis. It has been associated with the use of high-dose cyclophosphamide $(>50 \mathrm{mg} / \mathrm{kg})$, whereas it is regarded as a rare event with administration of low-dose cyclophosphamide $(<20 \mathrm{mg} / \mathrm{kg})$. Here we report 2 cases of breast cancer patients who developed severe symptomatic 
Baker et al.: Cyclophosphamide-Induced Severe Acute Hyponatremic Encephalopathy in Patients with Breast Cancer: Report of Two Cases

hyponatremia after receiving TC or AC. Both TC and AC are widely used in adjuvant and metastatic settings to treat breast cancer, and the dose of cyclophosphamide in these regimens is considered 'low'. Although it is always difficult to indict a particular drug after the administration of multiple chemotherapeutic agents and premedication, the temporal relationship of severe hyponatremia secondary to cyclophosphamide appears to be in line with that previously reported in the medical literature [1-3]. Moreover, the fact that retreatment with docetaxel or doxorubicin alone with exactly the same premedications did not induce hyponatremia or mental status change lends further support for this hypothesis.

Hyponatremia is a well-known complication in cancer therapy. One of the first reports of chemotherapy-induced hyponatremia by vincristine dates back to 1966 [4]. After this episode, cases have been reported in association with vinca alkaloids, platinum compounds, alkylating agents, immunomodulators, and monoclonal antibodies. However, the exact mechanism is largely unknown for most of these drugs.

Physiologically, antidiuretic hormone (ADH) is produced in the posterior pituitary gland in response to an increased plasma sodium concentration in order to retain water. The SIADH secretion is characterized by either a substantial release of ADH in the absence of stimuli or by the enhanced action of ADH on the kidneys [5]. Drug-induced SIADH can occur due to either or both mechanisms. Cyclophosphamide was first demonstrated to produce an antidiuretic hormone-like effect to retain water in 1974 [6]. SIADH was proposed as the mechanism for fatal hyponatremia in a patient having received cyclophosphamide infusion in 1979 [7]. More recent studies suggest a direct toxic effect of cyclophosphamide or its metabolites on renal collecting tubules and/or an antidiuretic hormone-like activity of cyclophosphamide metabolites [8]. Thus, development of SIADH has been accepted as one of the mechanisms for cyclophosphamide-induced hyponatremia. In the first case, the patient's urine osmolality was high (not available in the second case), which highly suggested SIADH.

Initially it was thought that only a high dose of cyclophosphamide $(>40 \mathrm{mg} / \mathrm{kg})$ could induce hyponatremia. However, low-dose cyclophosphamide $(<20 \mathrm{mg} / \mathrm{kg})$, including doses $<10 \mathrm{mg} / \mathrm{kg}$, has been noted to cause this potentially serious adverse event [4, 8-11].

A retrospective analysis of 112 treatment episodes including 84 patients (42 lupus nephritis and 42 non-Hodgkin's lymphoma) demonstrated cyclophosphamide-induced hyponatremia in 15 individual treatments (13.4\%) involving 12 patients $(14.3 \%)$, although none of the cases had severe hyponatremia [1]. A MEDLINE search found two other reports associating low-dose cyclophosphamide with symptomatic hyponatremia in breast cancer patients [2, 3]. In both cases, the patients received standard regimens, either AC or FEC (5fluorouracil, epirubicin, and cyclophosphamide), developed generalized seizure due to severe hyponatremia as our second patient, and recovered after slow correction of hyponatremia.

Symptomatology of hyponatremia is usually dependent on the duration of onset and the sodium level. It is usually asymptomatic if serum sodium is $130-135 \mathrm{mmol} / \mathrm{l}$. Nausea and malaise can occur when the serum sodium falls to $125-130 \mathrm{mmol} / \mathrm{l}$. Headaches, nausea, vomiting, muscle cramps, restlessness, disorientation, and depressed reflexes can be seen if the serum sodium falls below $125 \mathrm{mmol} / \mathrm{l}$. If severe hyponatremia (serum sodium levels $<120 \mathrm{mmol} / \mathrm{l}$ ) develops over a short period of time, neurological symptoms such as coma, permanent brain damage, even brain stem herniation or respiratory arrest may occur. In both cases, our patients presented with symptomatic encephalopathy, reflecting the severity of hyponatremia of acute onset.

Hyponatremia can be associated with different volume states: hypovolemia, euvolemia or hypervolemia, and therefore an accurate assessment of the patient's volume status is critical to determine the appropriate treatment strategy. Hypovolemic patients may present 
Baker et al.: Cyclophosphamide-Induced Severe Acute Hyponatremic Encephalopathy in Patients with Breast Cancer: Report of Two Cases

with orthostatic hypotension, tachycardia, and dry mucous membrane (as in case 1), whereas hypervolemic patients may present with edema or ascites. A urine sodium level $>30$ $\mathrm{mEq} / \mathrm{l}$ in the absence of diuretic use or renal disease is supportive of an euvolemic state (as in case 2) [12].

The treatment of hyponatremia depends largely on its onset (acute vs. chronic), etiology (volume status), and symptomatology. Hypovolemic hyponatremic patients should be treated with isotonic saline (as in case 1). Correction of sodium in acute hyponatremia should not exceed 1 to $2 \mathrm{mmol} / \mathrm{h}$ and $8 \mathrm{mmol} /$ day on any given day of treatment. The goal should be to raise the sodium to a safe level $(>120 \mathrm{mmol} / \mathrm{l})$ rather than complete normalization of the level. After a safe level is achieved, conservative measures such as fluid restriction can be utilized. For treatment of symptomatic acute hyponatremia in euvolemic or hypervolemic patients, hypertonic saline (3\% normal saline) can be used (as in case 2) [13].

\section{Conclusion}

In summary, we report 2 cases of acute hyponatremic encephalopathy induced by lowdose cyclophosphamide. Clinicians should be aware of this complication in order to make a correct diagnosis and provide appropriate treatment.

\section{References}

1 Lee YC, Park JS, Lee CH, et al: Hyponatraemia induced by low-dose intravenous pulse cyclophosphamide. Nephrol Dial Transplant 2010;25:1520-1524.

-2 Hwang SB, Lee HY, Kim HY, et al: Life-threatening acute hyponatremia with generalized seizure induced by low-dose cyclophosphamide in a patient with breast cancer. J Breast Cancer 2011;14:345-348.

-3 Bruining DM, van Roon EN, de Graaf H, et al: Cyclophosphamide-induced symptomatic hyponatraemia. Neth J Med 2011;69:192-195.

4 Berghmans T: Hyponatremia related to medical anticancer treatment. Support Care Cancer 1996;4:341350 .

5 Belton K, Thomas SH: Drug-induced syndrome of inappropriate antidiuretic hormone secretion. Postgrad Med J 1999;75:509-510.

6 Moses AM, Miller M: Drug-induced dilutional hyponatremia. N Engl J Med 1974;291:1234-1239.

7 Harlow PJ, DeClerck YA, Shore NA, et al: A fatal case of inappropriate ADH secretion induced by cyclophosphamide therapy. Cancer 1979;44:896-898.

8 Webberley MJ, Murray JA: Life-threatening acute hyponatraemia induced by low dose cyclophosphamide and indomethacin. Postgrad Med J 1989;65:950-952.

9 Koo TY, Bae SC, Park JS, et al: Water intoxication following low-dose intravenous cyclophosphamide. Electrolyte Blood Press 2007;5:50-54.

-10 Salido M, Macarron P, Hernandez-Garcia C, et al: Water intoxication induced by low-dose cyclophosphamide in two patients with systemic lupus erythematosus. Lupus 2003;12:636-639.

11 Park S, Kim W, Choi HY, Yoon JH, et al: Severe symptomatic hyponatremia caused by low dose oral cyclophosphamide: a case report. The Korean Journal of Nephrology 2011;30:647-650.

12 Castillo JJ, Vincent M, Justice E: Diagnosis and management of hyponatremia in cancer patients. Oncologist 2012;17:756-765.

13 Biswas M, Davies JS: Hyponatraemia in clinical practice. Postgrad Med J 2007;83:373-378. 